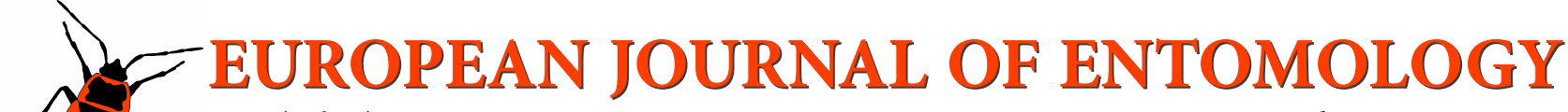 \\ ISSN (online): 1802-8829 \\ http://www.eje.cz \\ Eur. J. Entomol. 115: 602-613, 2018 \\ doi: 10.14411/eje.2018.057 \\ ORIGINAL ARTICLE
}

\section{Identification and characterization of doublesex from the pumpkin fruit fly, Bactrocera tau (Diptera: Tephritidae)}

\author{
ThANASEt THONGSAIKLAING ${ }^{1,2,3}$, Hataichanok PASSARA ${ }^{4}$, MINGKWAN NIPITWATHANAPHON 5 \\ and LERTLUK NGERNSIRI ${ }^{5, *}$
}

\author{
${ }^{1}$ Center of Agricultural Biotechnology, Kasetsart University, Kampaengsaen Campus, NakonPathom 73140, Thailand; \\ e-mail: thanaset_th@hotmail.com \\ ${ }^{2}$ Center for Excellence on Agricultural Biotechnology: (AG-BIO/PERDO-CHE), Bangkok 10900, Thailand \\ ${ }^{3}$ Division of Animal Science, Faculty of Agriculture, Princess of Naradhiwas University, Narathiwat 96000, Thailand \\ ${ }^{4}$ Interdisciplinary Graduate Program in Genetic Engineering, Kasetsart University, Bangkok, Thailand; \\ e-mail: hataichanokP12@gmail.com \\ ${ }^{5}$ Department of Genetics, Faculty of Science, Kasetsart University, Bangkok, Thailand; e-mails: mingkwan.n@ku.th, \\ lertlukngernsiri@gmail.com
}

Key words. Diptera, Tephritidae, doublesex, Bactrocera tau, pumpkin fruit fly, sex determination

\begin{abstract}
The sex determination cascades in insects are diversified at the top of the cascade, where different primary molecular signals are employed, while at the bottom of the cascades, particularly the doublesex genes, are highly conserved. Here, we identified the doublesex ortholog (Btau-dsx) of Bactrocera tau, a pumpkin fruit fly, and found that Btau-dsx is composed of six exons and five introns with an additional short " $m$ " exon located in the second intron. Btau-dsx is different from its orthologs in most dipteran insects: Its pre-mRNA is sex-specifically spliced to yield three (two male and one female) instead of two transcript variants. The two deduced proteins produced by the male-specific transcripts are a functional (Btau-DSX $\left.{ }^{\mathrm{M} 1}\right)$ and a truncated (Btau-DSX ${ }^{\mathrm{M} 2}$ ) protein, while the female-specific transcript produces the functional Btau-DSXF protein. These three proteins contain all conserved domains except Btau-DSX ${ }^{\mathrm{M} 2}$ which has no OD2 domain. The female-specific transcript is detected in both fertilized and unfertilized eggs and in both somatic and germ cells of the adult females, while the male-specific transcript is detected only in fertilized eggs and in the abdominal tissues and testes of adult males. The presence of the Btau-dsx ${ }^{M 1}$ transcript in fertilized eggs at the early syncytium stage suggests that in $X Y$ embryos, the $Y$-linked $M$ factor gene may function quite soon after fertilization to alter the splicing pattern of Btau-dsx pre-mRNA from the female-specific to the male-specific mode. Injection of Btau-dsx ${ }^{F}$ dsRNA into recently emerging females can reduce the expression of vitellogenin (Btau- $\mathrm{Vg}$ ) and causes some defects in the ovaries, indicating that Btau-dsx F works upstream of Btau-Vg.
\end{abstract}

\section{INTRODUCTION}

The most clearly known mechanism of sex determination among insects is obtained from studies of Drosophila melanogaster, whose sex determination cascade contains four key genes, sex lethal $(S x l)$, transformer (tra), transformer2 (tra2) and doublesex ( $d s x)$. These genes function in a hierarchical order, from the top one to the bottom gene (Cline, 1993; Bopp et al., 2014). The uppermost gene, $S x l$, is ON or OFF depending on the number of X chromosome. In XX flies, X-linked signaling elements (XSEs) act as a primary signal to activate $S x l$ and induce this gene to produce SXL protein. In XY flies, the concentration of XSEs is insufficient to activate $S x l$ expression and therefore the SXL protein is not produced. When Sxl protein is present, the downstream gene tra produces the female-specific pro- tein, TRA ${ }^{\mathrm{F}}$, which combines with TRA2, a non sex-specific protein, generating the TRA/TRA2 complex protein. This protein complex regulates $d s x$ splicing in the female mode to produce $\mathrm{DSX}^{\mathrm{F}}$, which directs female embryonic development. In XY embryos that do not have the SXL protein, both tra and $d s x$ genes produce male-specific proteins, $\mathrm{TRA}^{\mathrm{M}}$ and $\mathrm{DSX}^{\mathrm{M}}$, which induce embryos to develop as male flies (Baker \& Wolfner, 1988; Burtis \& Baker, 1989; Hoshijima et al., 1991; Salz \& Erickson, 2010).

Sex determination cascades of other insects do, however, seem to be different from that of Drosophila. In tephritid insects for example, the $S x l$ genes identified from Ceratitis capitata and Bactrocera oleae do not function in sex determination, although their $S x l$ sequences are highly similar to that of Drosophila (Saccone et al., 1998; Lagos et al.,

\footnotetext{
* Corresponding author; e-mail: lertlukngernsiri@gmail.com
} 
2005). Instead, the maternal tra genes identified in several tephritid insects are suggested to be the primary signals promoting embryonic sex fate. In XX embryos, functional TRA protein $\left(\mathrm{TRA}^{\mathrm{F}}\right)$ and TRA2 protein are both maternally provided and form a maternal TRA/TRA2 complex, ensuring via an autoregulation feedback loop that all zygotic tra pre-mRNA is spliced into the female-specific mode (Pane et al., 2002; Verhulst et al., 2010). $\operatorname{tra}^{F}$ mRNA produces the $\mathrm{TRA}^{\mathrm{F}}$ protein that, in turn, regulates female-specific splicing of $d s x$ in order to produce the female specific protein, $\mathrm{DSX}^{\mathrm{F}}$. In the presence of $\mathrm{DSX}^{\mathrm{F}}, \mathrm{XX}$ embryos develop as females. In $\mathrm{XY}$ embryos, the presence of $\mathrm{M}$ factors on $\mathrm{Y}$ chromosomes was suggested to turn off the tra autoregulatory feedback loop and, therefore, in the absence of TRA, $d s x$ produces the male-specific protein, $\mathrm{DSX}^{\mathrm{M}}$, promoting male development of XY embryos (Hoshijima et al., 1991; Lagos et al., 2007; Concha \& Scott, 2009; Salvemini et al., 2009; Sarno et al., 2010).

Besides dipteran insects, sex determination has been studied in seven insect orders (Price et al., 2015) including Lepidoptera (Ohbayashi et al., 2001; Shukla \& Nagaraju, 2010), Hymenoptera (Cho et al., 2007; Oliveira et al., 2009) and Coleoptera (Shukla \& Palli, 2012; Ito et al., 2013). The studies of sex determination in all insect species have thus far revealed that the primary signals initiating the sex determination cascade seem to vary, while genes, such as doublesex, functioning at the bottom of the cascades, are quite conserved. Doublesex ( $d s x)$, has been identified and characterized in a number of different insects. In most dipteran insects, $d s x$ genes are transcribed in both sexes but their pre-mRNAs are spliced in a sex-specific manner, producing one male- and one female-specific transcript that is translated into a $\mathrm{DSX}^{\mathrm{M}}$ protein in males and a $\mathrm{DSX}^{\mathrm{F}}$ protein in females (Baker \& Wolfner, 1988; Burtis \& Baker, 1989; Hediger et al., 2004; Lagos et al., 2005; Chen et al., 2008). DSX proteins are members of the doublesex/mab-3 related (Dmrt) family. Like other proteins belonging to the Dmrt family, DSX proteins have two conserved functional domains, an N-terminal DNA binding domain (DM or OD1 domain) and a C-terminal dimerization domain (DSX dimer or OD2 domain). OD1 is a common domain found in both $\mathrm{DSX}^{\mathrm{M}}$ and $\mathrm{DSX}^{\mathrm{F}}$, while the $\mathrm{C}$-terminal regions of the OD2 domain are different in the two proteins and direct an embryo containing $\mathrm{DSX}^{\mathrm{M}}$ towards the male sex fate or an embryo containing $\mathrm{DSX}^{\mathrm{F}}$ towards the female sex fate.

$d s x$ genes have been identified and characterized in many tephritid insects such as Bactrocera tryoni (Shearman \& Frommer, 1998), Anastrepha oblique (Ruiz et al., 2005), B. oleae (Lagos et al., 2005), Ceratitis capitata (Saccone et al., 2008), B. dorsalis (Chen et al., 2008; Permpoon et al., 2011), B. jarvisi (Morrow et al., 2014), and B. correcta (Permpoon et al., 2011). The structures of Bactrocera and Ceratitis $d s x$ genes are similar since they are composed of six exons and five introns, while Anastrepha $d s x$ genes are instead composed of four exons and three introns. However, in all cases $d s x$ genes are alternatively spliced in a sex-specific manner, producing one male $\left(\mathrm{DSX}^{\mathrm{M}}\right)$ and one female-specific $\left(\mathrm{DSX}^{\mathrm{F}}\right)$ protein.
The pumpkin fruit fly, Bactrocera tau (Walker), is an important pest widely distributed throughout South East Asian countries including Thailand (Chen, 2001). The sex determination genes have been suggested as potential targets for creating genetic sexing strains or for RNAi technology used in the pest management programmes (Dafa'alla et al., 2010; Whyard et al., 2015; Leftwich el al., 2016). In this study, we identify and characterize the $d s x$ homologue of B. tau and find that the structure of Btau-dsX is the same as that of other Bactrocera $d s x$ genes except for the existence of a short " $\mathrm{m}$ " exon located in the second intron. Interestingly, we detect the male-specific Btau-ds $x^{M 1}$ mRNA in fertilized embryos just after egg-laying. We also examine the function of Btau-dsx in regulating the downstream target gene, vitellogenin, by using adult abdominal double-strand RNA injection. We hope, therefore, that in the future the Btau-dsx gene may be utilized as a means to control the dispersion of this insect species.

\section{MATERIAL AND METHODS}

\section{Insect culturing}

Pumpkin fruit flies, Bactrocera tau, were kindly provided by Prof. Dr. Sangvorn Kitthawee, Department of Biology, Faculty of Science, Mahidol University. Flies were cultured on artificial food, and kept at $25^{\circ} \mathrm{C}, 70 \%$ relative humidity, and with a photoperiod of $12 \mathrm{~L}: 12 \mathrm{D}$. Cucumbers were used for egg collection.

\section{Cloning and sequencing of Btau-dsx}

Total RNA was individually extracted from the 15-day-old adult females and males separately using TRIzol reagent (Life Technologies, Carlsbad, CA, USA). One unit of DNase I per mg of RNA was added to the total RNA obtained. One microgram of total RNA was used for synthesis of the first cDNA strand of Btau-dsx using the Revert Aid First Strand cDNA Synthesis Kit (Thermo Fisher Scientific, Waltham, MA, USA) and following the manufacture's protocol. Primers were designed based on Bactrocera $d s x$ sequences available in the NCBI database. The first cDNA strand was used as a template to amplify the Btau-dsx gene with primers BtdsxF and BtdsxR (Table 1) under the following conditions: preheating at $94^{\circ} \mathrm{C}$ for $3 \mathrm{~min}$, followed by 35 cycles of denaturation at $94^{\circ} \mathrm{C}$ for $30 \mathrm{~s}$, annealing at $55^{\circ} \mathrm{C}$ for $30 \mathrm{~s}$ and extension at $72^{\circ} \mathrm{C}$ for $1 \mathrm{~min}$ : with a final extension at $72^{\circ} \mathrm{C}$ for 10 min. The PCR product was cloned in pGEM $^{\mathrm{T}}$ Easy plasmid vector (Promega, Madison, WI, USA) and sent to Macrogen, Seoul, Korea, for sequencing. The BLAST program (NCBI) was used to check that the sequence obtained from Marcrogen was that of the $d s x$ gene.

To obtain the full-length Btau-dsx cDNA sequence, both 5' and 3' Rapid Amplifications of cDNA Ends (RACE) were conducted using the smart cDNA Amplification Kit (Clontech, Mountain View, CA, USA) according to manufacturer's instructions and the gene-specific primers, BtdsxR5'RACE for $d s x$ ' ${ }^{\prime}$ RACE and BtdsxF3'RACE for $d s x$ 3' RACE (Table 1). The nested PCR for the $d s x$ 5' RACE was performed using the same kit and a specific primer, BtdsxnestR5'RACE (Table 1). The 5' RACE PCR and 3'RACE PCR fragments were cloned and sequenced at Macrogen. All obtained PCR sequences were assembled to get the full length Btau-dsx cDNA sequence using the CAP3 Sequence Assembly Program (http://pbil.univ-lyon1.fr/cap3.php).

\section{Identification of the Btau-dsx genomic structure}

The genomic DNA sequence of $B$. cucurbitae (NW_011863834.1) available in the NCBI database was used 
Table 1. Primers used in this study.

\begin{tabular}{|c|c|}
\hline Primer name & Sequence primer 3'-5' \\
\hline BtdsxF & GGC-CAC-AAA-CGC-TAT-TGT-AAA-TAC-CCG \\
\hline BtdsxR & AAT-GGC-ATC-ATC-TCC-CAT-GGA-TAT-CG \\
\hline BcdsxR5'RACE & GGG-CAA-TAC-CGC-TCC-CGA-AGT-GGA \\
\hline BcdsxF3'RACE & GCA-GAC-AGC-ATT-GAG-GCG-GGC-ACA-G \\
\hline UMP & CTA-ATA-CGA-CTC-ACT-ATA-GGG-CAA-GCA-GTG-GTA-TCA-ACG-CAG-AGT \\
\hline BtdsxnestR5'RACE & GCG-GGG-GAG-GAG-ATA-GAG-CTG-CCA \\
\hline UMP short & CTA-ATA-CGA-CTC-ACT-ATA-GGG-C \\
\hline dsx-dsRNAi2-F & TAA-TAC-GAC-TCA-CTA-TAG-GGA-GAC-CAC-TAA-GTG-TGA-ATA-CCA-GCA-TTT-CTG \\
\hline dsx-dsRNAi2-R & TAA-TAC-GAC-TCA-CTA-TAG-GGA-GAC-CAC-CCT-AAA-ATA-TTT-TTT-ATT-ATT-GTA \\
\hline BttradsRNA-F & GTA-ATA-CGA-CTC-ACT-ATA-GGG-GGA-GAG-TGA-AGT-TGG-ACC-CA \\
\hline dsDsRed_F_T7 & CCG-GAT-CCT-AAT-ACG-ACT-CAC-TAT-AGG-GCG-GTG-CTT-GTC-AAT-GCG-GTA-AGT-G \\
\hline dsDsRed_R_T7 & CCG-GAT-CCT-AAT-ACG-ACT-CAC-TAT-AGG-GCG-CTC-GAG-ATC-TGA-CAA-TGT-TCA-G \\
\hline BtdsxFF - & TTG-CCC-ATA-TCG-GTA-GTT-GGC \\
\hline R-dsxF & TCA-TCC-GCA-TTG-CCT-TGT-CGT-AC \\
\hline R-dsxM & CGG-CTG-CTG-GCG-GTG-ACA-TC \\
\hline BTdsxF-qRT & TGG-TTT-CGG-AGG-ATA-GTT-G \\
\hline BTdsxR-qRT & GTA-TCT-GCA-GAA-CCC-TTT-GT \\
\hline BTvgF-qRT & AGC-GTT-TAG-CTC-TCA-TCG \\
\hline BTvgR-qRT & GAT-GGC-GTG-TAC-CCA-TTC \\
\hline BTactF-qRT & GAG-GAG-CAC-CCA-GTT-CTG-C \\
\hline BTactR-qRT & GTA-GCC-GCG-CTC-AGT-CAG \\
\hline BTactF & GAG-GAG-CAC-CCA-GTT-CTG-C \\
\hline BTactR & GTA-GCC-GCG-CTC-AGT-CA \\
\hline
\end{tabular}

as a template for alignment with all Btau-dsx full-length cDNA sequences using ClustalW2 version 2.0.10 to get the number of exons and introns.

\section{Multiple alignment and phylogenetic analysis}

Full length Btau-dsx cDNA sequences were translated into the deduced protein sequences using the Expasy-Translation tool (web.expasy.org/translate/) and were then aligned with a selection of insect Dsx protein sequences (Table S1) available in the NCBI database using clustalW2 version 2.0.10 to highlight sequence identities. Phylogenetic trees were constructed using the neighbour-joining method in MEGA version 7.0.21 (Kumar et al., 2016). The reliability of clusters within the tree was evaluated based upon 1000 bootstrap replications.

\section{Expression analysis using RT-PCR}

Total RNA was extracted from both fertilized and unfertilized eggs of B. tau at several development stages (0.5-24 h), from whole bodies of adult males and females 2-10 days old, and from some tissues (heads, thoraxes, abdomens, ovaries and testes) of adult males and females at 15, 20 and 25 days post-eclosion, using GenUP ${ }^{\mathrm{TM}}$ Total RNA Kit (Biotechrabbit, Hennigsdorf, Germany) following the manufacture's instruction. Three micrograms of total RNA was reverse-transcribed (Reverse transcription System Kit; Promega). Multiplex RT-PCRs were performed using these cDNAs as templates and three primers, BtdsxFF, R-dsxF and R-dsxM (Table 1), for amplifying $d s x^{F}$ and $d s x^{M}$ transcripts. The $\beta$-actin gene was used as a reference gene and amplified with primers $\beta$-actinF and $\beta$-actinR (Table 1). Amplification was carried out in a total volume of $50 \mu \mathrm{l}$, including $1 \mu \mathrm{g}$ of cDNA, $2 \mu \mathrm{l}$ of each primer $(10 \mathrm{mM}), 1 \mu \mathrm{l}$ of dNTP $(10 \mathrm{mM}), 0.5 \mu \mathrm{l}$ of Taq DNA polymerase, and $33.5 \mu \mathrm{l}$ of distilled water. The PCR reaction was performed at $95^{\circ} \mathrm{C}$ for $2 \mathrm{~min}$, followed by 30 cycles of $95^{\circ} \mathrm{C}$ for $30 \mathrm{~s}, 60^{\circ} \mathrm{C}$ for $30 \mathrm{~s}$, and $72^{\circ} \mathrm{C}$ for $1 \mathrm{~min}$, with a final step of $5 \mathrm{~min}$ at $72^{\circ} \mathrm{C}$. The PCR products were analyzed on $1 \%$ agarose gels.

\section{Preparation of double-stranded RNAs and microinjection procedures}

The region common to all $d s x$ genes containing the OD1 domain sequence (752 bp long) was amplified with primers dsxdsRNAi2-F and dsx-dsRNAi2-R (Table 1) and cloned into a
pGEM ${ }^{\mathrm{T}}$ Easy plasmid vector. This clone was used as a template for preparing Btau-dsx dsRNAs using a MEGA script T7 kit (Ambion, Foster City, CA, USA), according to the manufacturer's instructions. As a control, $562 \mathrm{bp}$ of the DsRed gene was amplified using primers dsDsRedF and dsDsRedR with the amplification product being used as a template for synthesis the DsRed dsRNA using the same protocol (Table 1). One microgram per microliter of either Btau-dsx dsRNA or DsRed dsRNA was injected into the third abdominal segment of each adult female within $1 \mathrm{~h}$ of eclosion using a $10 \mu \mathrm{l}$ Hamilton microsyringe. Two females were collected on each of the $2^{\text {nd }}, 4^{\text {th }}, 6^{\text {th }}, 8^{\text {th }}$ and $10^{\text {th }}$ day post-injection and kept at $-80^{\circ} \mathrm{C}$ for analyzing the expression of Btau-dsx and Btau-Vitellogenin genes. Some remaining females were dissected on the $10^{\text {th }}$ day post-injection, their ovaries were observed under an Olympus stereomicroscope and photographs were taken with a DP72 Olympus digital camera.

\section{Real-time PCR experiment}

Total RNA was separately extracted from whole bodies of all collected Btau-dsx or DsRed dsRNAs injected B. tau females using TRIzol reagent and following the manufacturer's protocol. Three micrograms of total RNA were reverse-transcribed to cDNAs using a RevertAid First Strand cDNA Synthesis Kit (Thermo Fisher Scientific). The expression of Btau-dsx and Btau- $V g$ genes were examined by qPCR experiments using the Mastercycler ${ }^{\circledR}$ ep realplex (Eppendorf Co., Ltd, Hamburg, Germany). qPCR reactions were performed using RBC ThermOne ${ }^{\mathrm{TM}}$ Real-Time Premix (with SYBR Green). The qPCR program for amplifying the cDNAs was one cycle of $94^{\circ} \mathrm{C}$ for $3 \mathrm{~min}$ and 40 cycles of $94^{\circ} \mathrm{C}$ for $30 \mathrm{~s}, 57^{\circ} \mathrm{C}$ for $30 \mathrm{~s}$ and $72^{\circ} \mathrm{C}$ for $30 \mathrm{~s}$, followed by melting curve analysis to detect a specific amplification product. The primers used for Real-time PCR were BTdsxF-qRT, BTdsxR-qRT (Table 1) for assaying the transcription level of Btau-dsx ${ }^{F}$, BTvgF-qRT, BTvgF-qRT and BTvgR-qRT (Table 1) for detecting the Btau-vitellogenin (Btau-Vg) gene and BTactFqRT and BTactR-qRT (Table 1) for detecting the expression of the $\beta$-actin gene. Each sample was analyzed in triplicate and normalized to the $\beta$-actin expression of $B$. tau as an internal control. Real-time data were calculated using the relative expression level by the $2^{-\triangle A C T}$ method (Livak \& Schmittgen, 2001).

Primers for amplifying the $\mathrm{Btau}-\mathrm{Vg}$ gene were designed using the sequences from a selection of insect vitellogenin genes avail- 


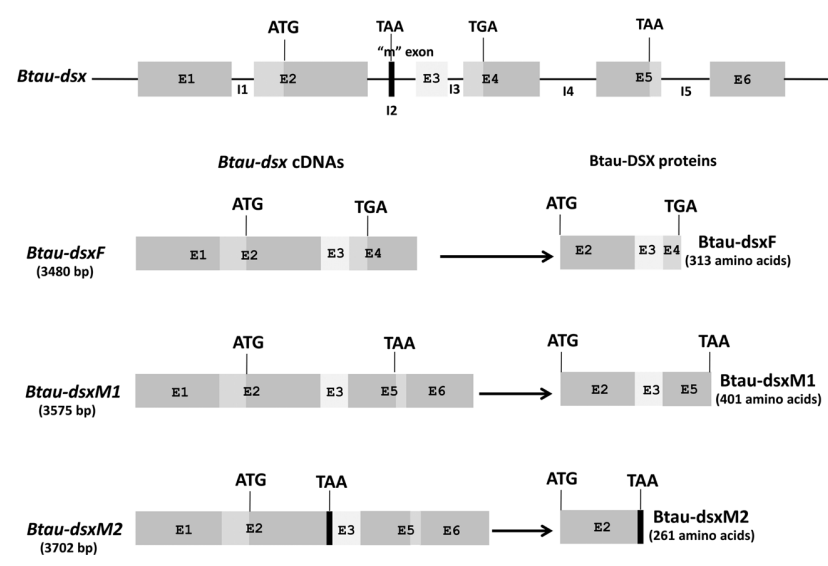

Fig. 1. Schematic representation of the genomic organization of the Btau-dsx gene, and Btau-dsx cDNAs and Btau-DSX proteins. Gray boxes (E1-E6) and straight lines (I1-I5) indicate exons and introns. The narrow black box indicates the $127 \mathrm{bp}$ sequence inserted in the second intron (I2).

able on the NCBI database. RT-PCR was performed using the B.tau female cDNA as a template and amplified with the designed primers. The obtained PCR fragment was cloned into a $\mathrm{pGEM}^{\mathrm{T}}$ easy vector and sent to Macrogen for sequencing. The obtained nucleotide sequence was blasted using the BLAST program (NCBI) to confirm that it was the sequence of the vitellogenin gene.

\section{RESULTS}

\section{Identification of the Btau-dsx gene structure and its transcript variants}

The structure of the Btau-dsx gene was identified by aligning the cDNA sequences of all Btau-dsx transcripts with the genomic sequence of the Bactrocera cucurbitae $d s x$ gene (NW_011863834.1). The result showed that Btau-dsx consisted of six exons and five introns, the same genomic structure as that of $D$. melanogaster and of other Bactrocera insects reported earlier (Fig. 1). The Btau- $d s x$ gene was transcribed in both sexes but its pre-mRNA was spliced in sex-specific manners, resulting in three transcripts: one female (Btau- $\left.d s x^{F}\right)$ and two male (Btau- $d s x^{M I}$ and Btau-dsx $\left.x^{M 2}\right)$. The female transcript consisted of four exons 1, 2, 3 and 4, while the male transcripts harbored five exons, 1, 2, 3, 5 and 6 (Fig. 1). However, only the BtaudsxM2 transcript contained an additional small exon (127 bp long, called the " $m$ " exon) located within the second intron. Alignment of these two male-specific transcripts with the genomic sequence of the B. cucurbitae $d s x$ gene revealed that this gene contained the " $\mathrm{m}$ " exon in the second intron. The full length cDNA sequence of Btau- $d s x^{F}$ (accession number: KP844899) was 3477 bp long and contained a $969 \mathrm{bp}$ ORF encoding a deduced protein of 322 amino acid residues. While, the complete cDNA sequences of Btau-dsx ${ }^{M 1}$ (accession number: KP844901) and Btau-dsx $x^{M 2}$ (accession number: KP844900) were 3575 and 3436 bp long, respectively. In the Btau- $d s x^{M 2}$ transcript, the $127 \mathrm{bp}$ " $\mathrm{m}$ " exon was located between exons 2 and 3, and contained a stop codon (TAA). Thus, the 786 bp long ORF of Btau-ds $x^{M 2}$ was quite short in comparison to Btau- $d s x^{M 1}$ (1206 bp) and encoded a short protein of 261 amino acid

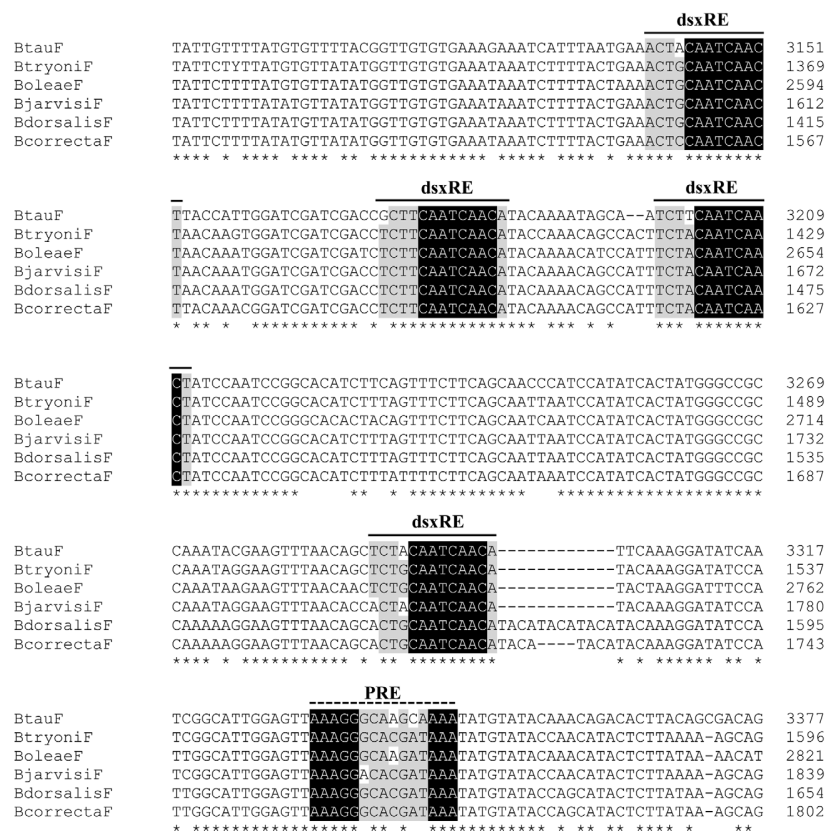

Fig. 2. Comparison of the 3 ' UTR of Bactrocera dsx female-specific transcripts show five conserved domains, four dsxRE elements (13 nucleotides) indicated by black lines above the domains and the purine-rich element (PRE) indicated by a dashed line above the corresponding domain, while white letters in black boxes show the identical eight nucleotide (CAATCAAC) sequences present in all Bactrocera dsxRE elements.

residues, while Btau- $d s x^{M 1}$ produced a deduced 401 amino acid functional protein.

The 3' UTR of the Btau-dsx $x^{F}$ transcript contained five conserved domains, four putative $13 \mathrm{nt} d s x$ repeat elements (dsxRE) and a purine rich element (PRE), followed by a poly (A) signal. Comparison of the Btau- $d s x^{F}$ mRNA sequence and other Bactrocera $d s x^{F}$ mRNA sequences available in the NCBI database indicated that the $d s x^{F}$ mRNA of Bactrocera species commonly contained four dsxRE elements, with an identical eight nucleotide sequence (CAATCAAC) present in all Bacterocera dsxRE elements (Fig. 2). Moreover, the PRE element of Btau- $d s x^{F}$ was composed of 15 nucleotides and 13 of the 15 nucleotides were purines, while other Bactrocera PRE elements contained 12 purine nucleotides (Fig. 2).

\section{Amino acid sequence alignment and phylogenetics}

The amino acid sequences of these three Btau-DSX proteins and those of some other insect DSX proteins available in the NCBI database were aligned using the clustalw2 program. The identity of Btau-DSX ${ }^{\mathrm{F}}$ to the two male-specific DSX proteins, Btau-DSX ${ }^{\mathrm{M} 1}$ and Btau-DSX ${ }^{\mathrm{M} 2}$, was 93.48 and $95.40 \%$, respectively, while the identity between Btau$\mathrm{DSX}^{\mathrm{M} 1}$ and Btau-DSX ${ }^{\mathrm{M} 2}$ was $95.40 \%$. The amino acid sequences of the three Btau-DSX proteins (at amino acid position 1-246) were identical at the $\mathrm{N}$ terminal (containing the DNA binding domains, OD1, at position 38-100) until the beginning of the oligomerization (OD2) domains. The three Btau-DSX proteins were different at their $\mathrm{C}$ terminals, where the OD2 domains and sex-specific regions were located. However, the common region of the OD2 domains 

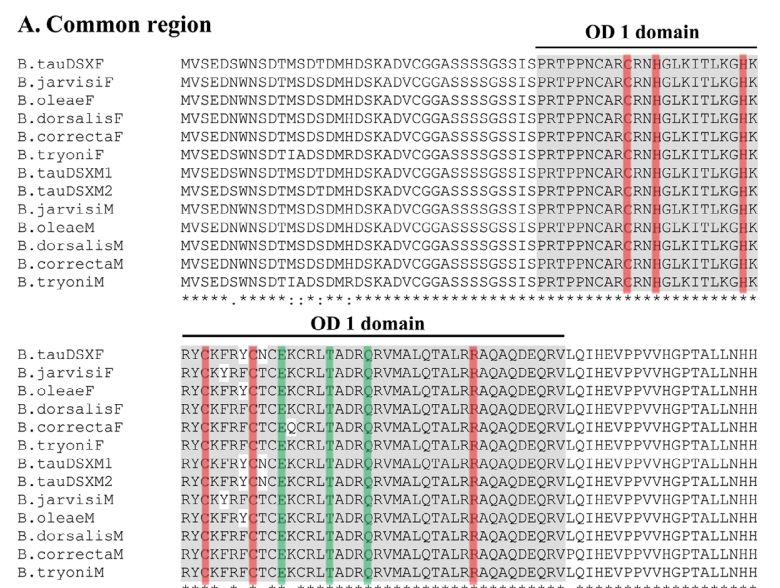

B.tauDSXF
B.jarvisiF
B.oleaef
B.dorsalisF
B.correctaF
B.tryoniF
B.tauDSXM1
B.taudSXM2
B. jarvisiM
B.oleaeM
B.dorsalisM
B.correctaM
B.tryoniM

B.tauDSXF
B.jarvisiF
B.oleae
B.dorsalisF
B.correctaF
B.tryoniF
B.tauDSXM1
B.taunSXM2
B.jarvisiM
B.oleaeM
B.dorsalisM
B.correctaM
B.tryoniM

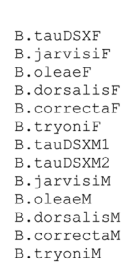
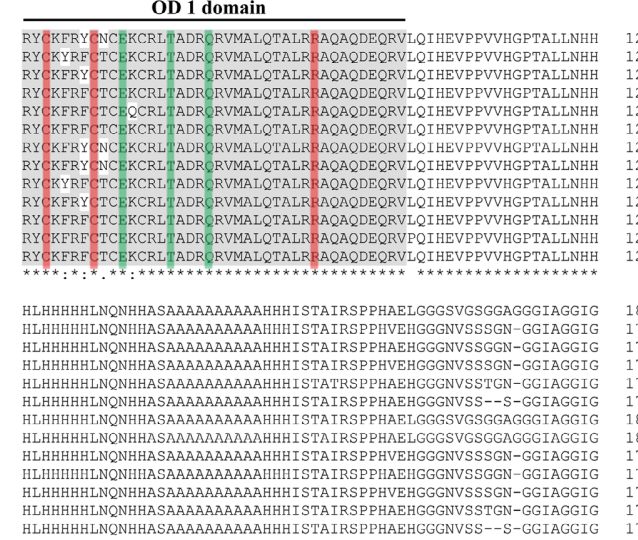

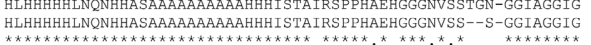

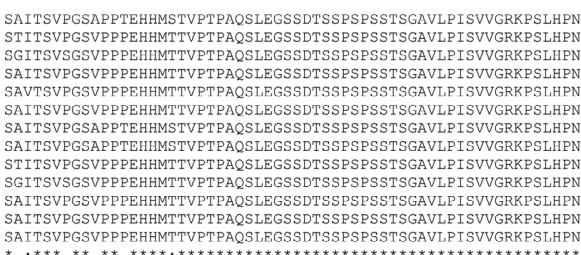

OD 2 domain

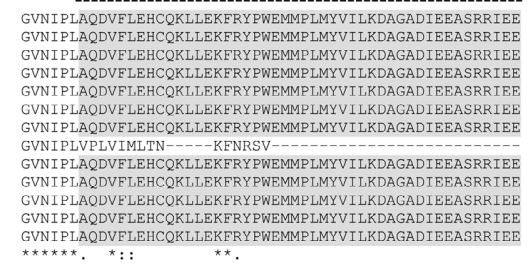

(46 amino acids at position 247-292) of Btau-DSX ${ }^{\mathrm{F}}$ and Btau-DSX ${ }^{\mathrm{M} 1}$ were also identical, while Btau-DSX ${ }^{\mathrm{M} 2}$ lacked the OD2 domain including the sex-specific region, indicating that it was a truncated protein. Alignment of these three Btau-DSXs with all other tephritid DSX proteins available in the NCBI database showed a percentage identity ranging from 85.17 to $95.95 \%$, while the identities to some DSX proteins of other insect orders were between 34.21 and $57.99 \%$. Comparison of all Bactrocera DSX proteins available in the NCBI database, including the three BtauDSX proteins, showed that the amino acid sequences at the $\mathrm{N}$-terminal regions were highly conserved. Furthermore, at the C-terminal regions, if Btau-DSX ${ }^{\mathrm{M} 2}$ was excluded, the amino acid sequences of the OD2 domains including the male-specific regions were completely identical, while the sequences of female-specific regions were also highly conserved (Fig. 3).

Moreover, the OD1 and the OD2 domains of $B$. tau DSX proteins (Btau-DSX ${ }^{\mathrm{F}}$ and Btau-DSX ${ }^{\mathrm{Ml}}$ ) were aligned with those of another 16 insect species available in the NCBI database. The alignment showed that at OD1 domains, 20 of 63 amino acid residues were conserved. The 20 conserved amino acids included six essential amino acid residues $(\mathrm{C}$,

\section{B. Female specific region}
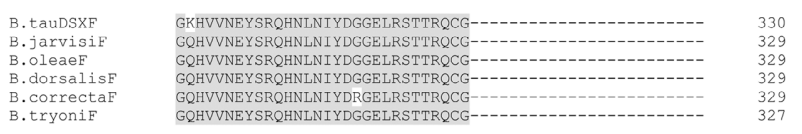

C. Male specific region

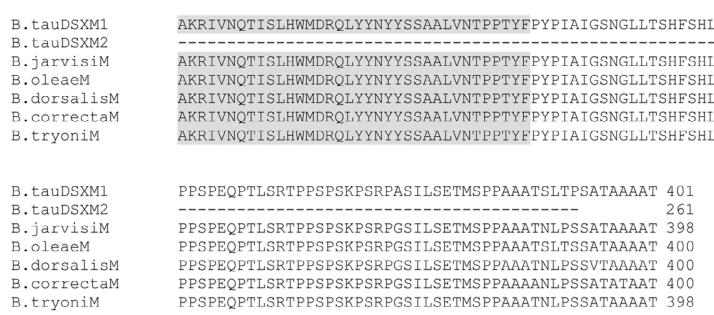

Fig. 3. Comparison of the amino acid sequences of BtauDSXM1, BtauDSXM2 and DSXF protein with other Bactroceran DSX proteins available in the NCBI database. (A) The two common regions, DNA binding domain (OD1) and oligomerization domain (OD2) are present in both male and female DSX proteins. The OD1 domain is labeled in gray and indicated by a black line above the domain and the OD2 domain is shaded in gray and indicated by a black dotted line above the domain. Six amino acids labeled with red are conserved to those of $D$. melanogaster in which they are required for DNA-binding activity and three amino acids labeled with green are specifically conserved to all insect OD1 domains. (B) The femalespecific region is labeled in gray. (C) The male-specific region is labeled in gray.

$\mathrm{H}, \mathrm{H}, \mathrm{C}, \mathrm{C}$ and $\mathrm{R}$ ) necessary for DNA-binding activity in D. melanogaster (Erdman \& Burtis, 1993) and three amino acid residues (E, T, Q) specific to the insect OD1 domains (Oliveira et al., 2009) (Fig. 4). In contrast, OD2 domains showed high variability, with only two positions out of 47 amino acid residues conserved (Fig. 4).

A phylogenetic tree was constructed using the deduced amino acid sequences of Btau-DSXs (Btau-DSX' ${ }^{\mathrm{F}}$, Btau$\mathrm{DSX}^{\mathrm{M} 1}$ and Btau-DSX ${ }^{\mathrm{M} 2}$ ) and the DSX proteins of other insects available in the NCBI database, by the neighborjoining method replicated 1000 times with bootstrap resampling. The tree topology showed that sequences of the DSX proteins could be used to separate insects at the order and genus levels. Tephritid insects were grouped in the same clade that was, using a classical taxonomy, separated into three subclades, namely Bactrocera, Anastrepha and Ceratitis (Fig. 5).

\section{Analysis of Btau-dsx gene expression patterns using RT-PCR}

Expression levels of the three sex-specific Btau-dsx transcripts at embryonic stages were assessed, this included both male and female embryos, since they could not be 


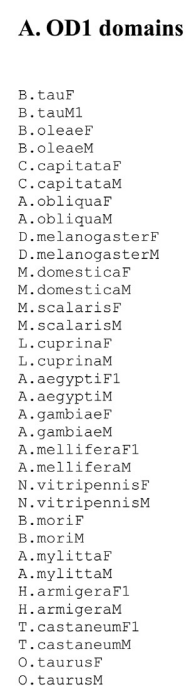

B. OD2 domains
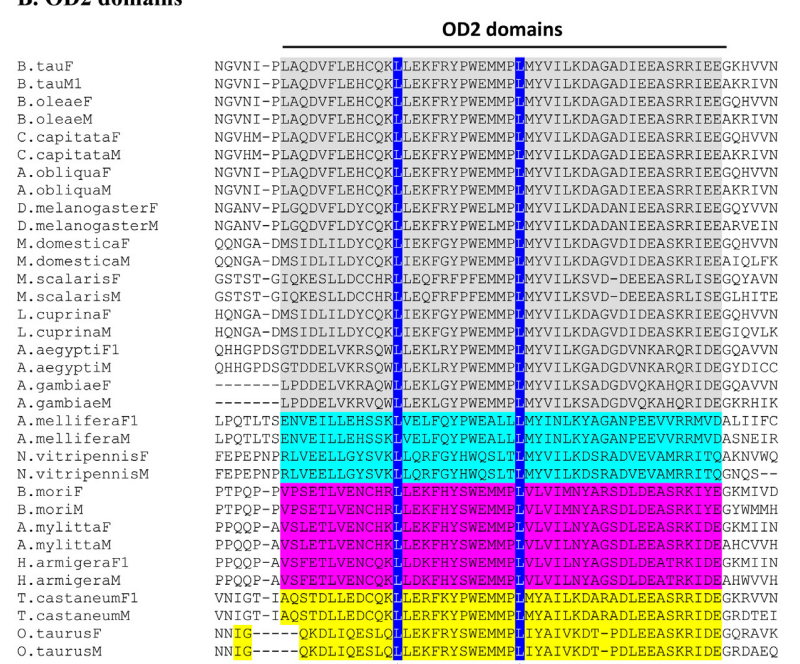

Fig. 4. Alignment of the OD1 and the OD2 domains of Btau-DSXs and some insect DSX proteins available in the NCBI database. (A) The OD1 domains. Amino acid residues labelled with red are essential for DNA-binding activity in $D$. melanogaster and the three amino acids labelled with green are specific to the insect OD1 domains. (B) The OD2 domains. Amino acid residues labelled with blue are conserved residues found in all insect DSX proteins present here.

separated from each other. The results showed that fertilized eggs after $0.5-24 \mathrm{~h}$ contained both Btau-dsx $x^{F}(295$ bp band) and Btau-dsx $x^{M 1}$ (487 bp band) transcripts but the

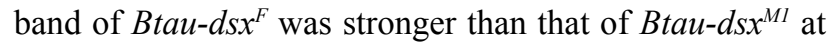
all stages detected (Fig. 6A). In unfertilized eggs at 0.5, 3,6 and $12 \mathrm{~h}$ after oviposition, only the Btau- $d s x^{F}$ transcript was detected, but this transcript disappeared at 24 h (Fig. 6B). In adult stages, the Btau- $d s x^{F}$ transcript was detected in adult females on each examination day $(2,4$, 6, 8 and 10 days post-eclosion). In adult males, the Btau$d s x^{M 1}$ transcript was detected in all examination days $(2$, $4,6,8$ and 10 days post-eclosion), while the Btau-ds $x^{M 2}$ transcript was detected only in adult males at 8 and 10 days post-eclosion (Fig. 6C). We found that Btau-dsx expression in some adult tissues of both sexes 15, 20 and 25 days after eclosion gradually increased and that the Btau-dsx $x^{F}$ tran-

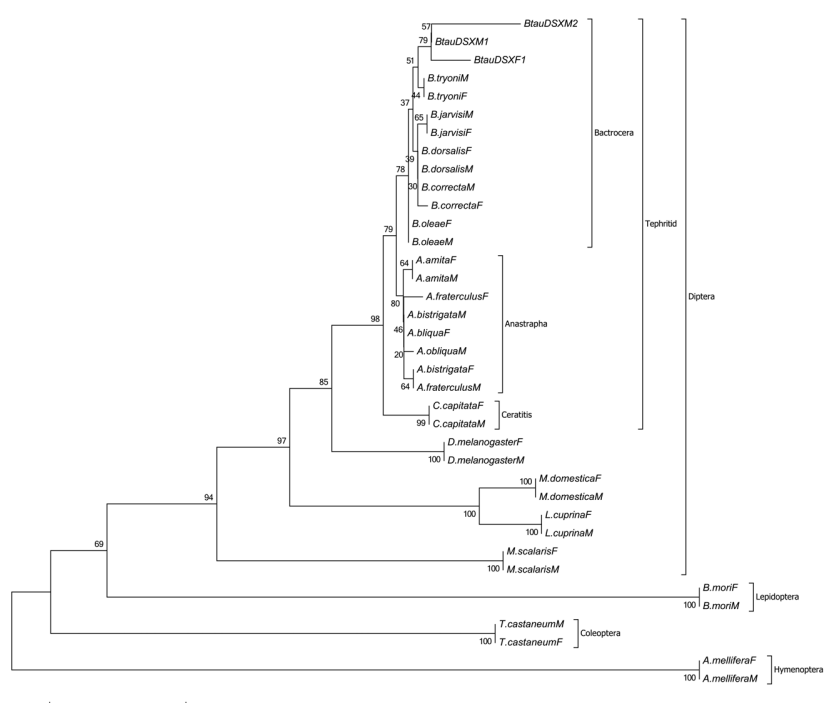

Fig. 5. Phylogenetic tree constructed using amino acid sequences of DSX proteins of a selection of insects available in the NCBI database using the neighbor-joining method in MEGA 7.0.21.

script was detected in all tissues examined (heads, thoraxes, abdomens and ovaries). Expression levels were similar in tissues from flies examined on the same day. In male tissues (heads, thoraxes, abdomens and testes), the Btau$d s x^{M 1}$ transcript was strongly detected only in abdomens and testes, while the Btau-dsx $x^{M 2}$ transcript was detected at comparatively very low levels (Fig. 6D).

\section{Functional analysis of Btau-dsx ${ }^{F}$ using RNA interference}

The Btau- $d s x^{F}$ transcript was only present in female flies. In this study, we reduced the quantity of Btau- $d s x^{F}$ transcripts in female flies by injecting early emerged females with Btau-dsx dsRNA. Ten days after injection, females were dissected and their ovaries were examined. The results showed that the ovaries of some injected females were mildly deformed: for example, eggs were shorter and their number was reduced, while the ovaries of females injected with DsRed dsRNA were relatively normal and were similar to those of uninjected females (Fig. 7). Moreover, the efficacy of Btau-dsx dsRNA in the reducing the levels of endogenous Btau-dsx $x^{\mathrm{F}}$ mRA was examined by quantitative real-time PCR (qPCR). Samples of females injected with Btau-dsx dsRNA were examined at 2, 4, 6, 8 and 10 days post-eclosion; the result showed that the levels of endogenous Btau-dsx $x^{F}$ mRNA were significantly lower than those of females injected with DsRed dsRNA $(P<0.01$, Student $t$-test) (Fig. 8) except at 2 and 10 day post-injection. Furthermore, we also examined the effect of Btau-dsx dsRNA on the expression of the Btau-vitellogenin (Btau$V g$ ) gene. In oviparous animals including insects, the $V g$ gene is expressed in females to produce a vitellogenin protein that is accumulated in the yolk of the eggs and is an essential nutrient for embryos during their development. We found that the level of Btau-Vg mRNA in females injected with Btau-dsx dsRNA was dramatically reduced compared to that of females injected with DsRed dsRNA $(P<0.01$, 

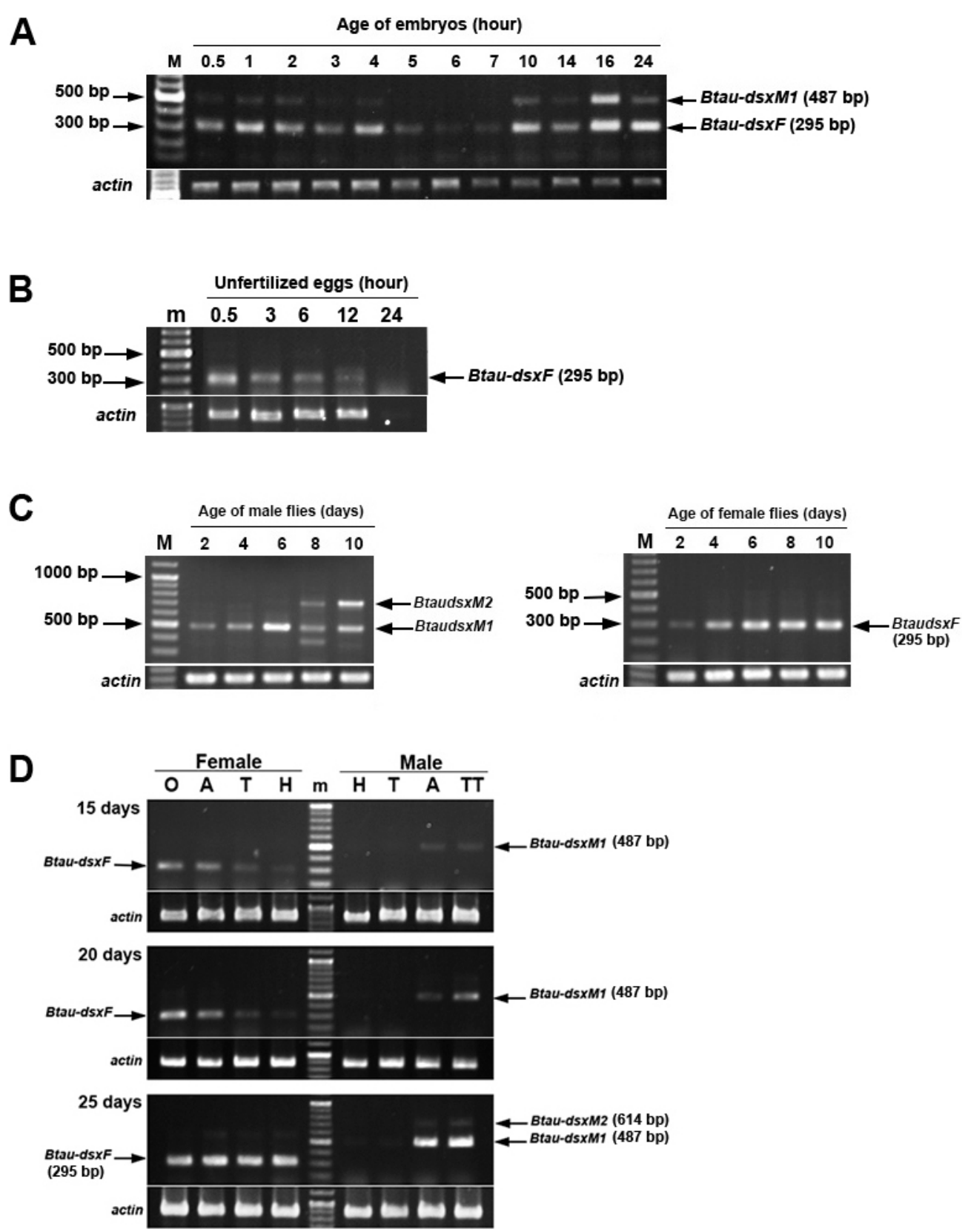

Fig. 6. The expression profiles of the Btau-dsx gene at several stages using RT-PCR. (A) 0.5-24 h fertilized eggs. (B) $0.5-24 \mathrm{~h}$ unfertilized eggs. (C) Adult males and females 2-10 days old. (D) Adult female and male tissues. $\mathrm{H}-$ head, $\mathrm{T}-$ thorax, A - abdomen, $\mathrm{O}-\mathrm{ovary}$, TT - testis.

Student $t$-test), indicating that Btau-dsx functions as an upstream gene regulating the expression of Btau- $\mathrm{Vg}$ (Fig. 9).

\section{DISCUSSION}

In this study, we identified the doublesex (Btau-dsx) gene from the pumpkin fruit fly, Bactrocera tau (Walker), an invasive insect pest distributed worldwide. The structure and function of Btau-dsx was similar to most dipteran $d s x$ genes, however Btau-dsx showed some different aspects from most dipteran and other Bactrocera $d s x$ genes such as the number of transcript variants, the presence of the " $m$ " exon, and the presence of the male-specific transcript at the early syncytial stages of fertilized eggs.

\section{The structure and transcript variants of the $B$. tau doublesex gene}

The structure of the Btau-dsx gene was found to be similar to that of D. melanogaster, M. domestica and Bactrocera insects. Btau-dsx consisted of six exons and five introns and was alternatively spliced in a sex-specific manner. The first three exons were common to both male and female transcript variants, while exon 4 was a female-specific exon, and exon 5 and 6 were male-specific (Burtis \& Baker, 1989; Lagos et al., 2005; Saccone et al., 2008). Similar to the $d s x$ genes of $M$. domestica and L. cuprina, Btau-dsx also contained the $127 \mathrm{bp}$ " $\mathrm{m}$ " exon located in the second intron between exon 2 and 3, and after splicing, the " $\mathrm{m}$ " exon remained only in one of the two male-specific transcripts, Btau-dsx $x^{M 2}$. The "m" exons of M. domestica 

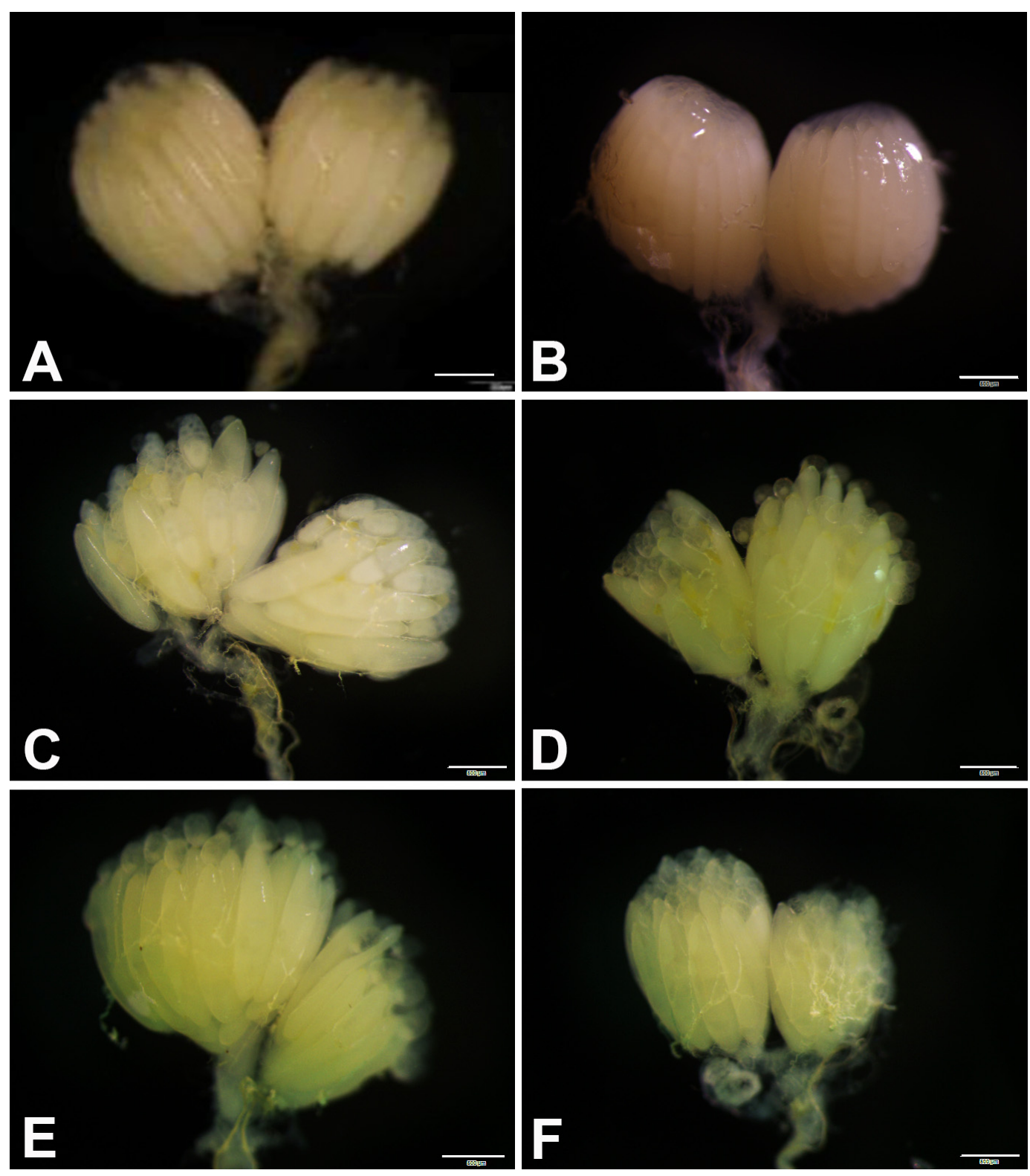

Fig. 7. Effect of Btau-dsx dsRNA on ovaries. (A) Ovary of an uninjected female. (B) Ovary of a female injected with DsRed dsRNA. (C-F) Ovaries of females injected with Btau-dsx dsRNA. Bars $=500 \mu \mathrm{m}$.

and L. cuprina $d s x$ genes were found to be 142 and $141 \mathrm{bp}$ long, respectively, and located in the intron between exon 3 and 4 (Hediger et al., 2004; Concha \& Scott, 2010). In $M$. domestica, after processing the $d s x$ pre-mRNA, the " $m$ " exon was present only in the male-specific and minor common transcript (Hediger et al., 2004). In fact, in Bactrocera insects, the "m" exon was also present in the second intron of the $d s x$ genomic region of Bactrocera cucurbitae, but was not found in that of Bactrocera oleae.

In contrast to most dipteran $d s x$ genes, Btau- $d s x$ was transcribed into three (one female and two male) instead of two (one male and one female) transcript variants (An et al., 1996; Shearman \& Frommer, 1998; Hediger et al., 2004; Lagos et al., 2005; Chen et al., 2008; Saccone et al., 2008; Permpoon et al., 2011; Morrow et al., 2014). The splicing of the $d s x$ pre-mRNAs to produce more than two transcript variants had been reported in many insect spe- cies, however, almost all additional $d s x$ transcripts are female-specific transcripts, such as two in Aedes aegypti (Salvemini et al., 2011), Apis mellifera (Cho et al., 2007), Bombyx mori (Ohbayashi et al., 2001), Antheraea mylitta (Shukla \& Nagaraju, 2010), three in Tribolium castaneum (Shukla \& Palli, 2012), and six in A. assama (Shukla \& Nagaraju, 2010). Two examples of a $d s x$ gene producing more than one male-specific transcript have been reported in two insect species, a Japanese rhinoceros beetle, Trypoxylus dichotomus, 6 (F) female-specific transcripts, and 2 (M) male-specific transcripts (Ito et al., 2013), and a fungus fly, Sciara ocellaris (2F and 2M) (Ruiz et al., 2015). Therefore, splicing of Btau-dsx pre-mRNA to produce two male-specific transcripts may be the first multiple malespecific $d s x$ pre-mRNA identified in tephritid insects.

In $D$. melanogaster, the 3 ' UTR of the $d s x$ female-specific exon (exon 4) harbored six 13 nt repeat elements (dsxRE) 


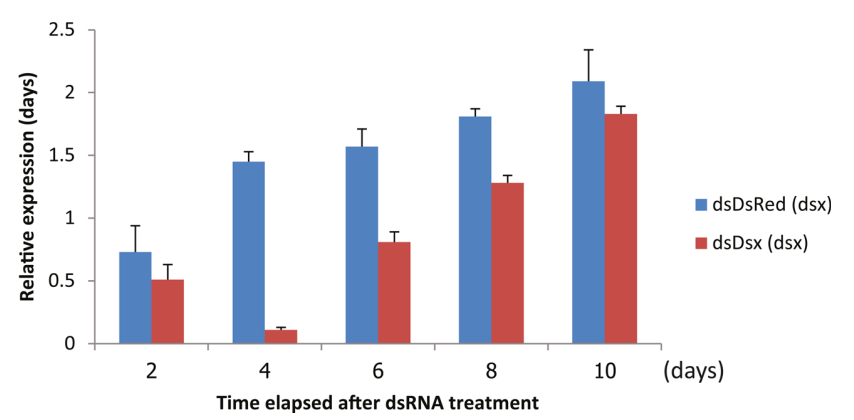

Fig. 8. Relative doublesex expression in females injected with either Btau-dsx dsRNA or DsRed dsRNA on 2, 4, 6, 8 and 10 days post-injection. Bars represent the mean \pm SD obtained from $q P C R$ experiment.

and a purine-rich element (PRE) that were the binding sites for the Tra/Tra2 protein complex. This protein complex is necessary for activation of the female-specific mode processing of $d s x$ pre-mRNA (Hoshijima et al., 1991; Inoue et al., 1992). Currently, these conserved domains have been identified in many dipteran $d s x$ female-specific transcripts including Btau-dsx $x^{F}$. The Btau- $d s x^{F}$ transcript harbors four 13 nt repeat elements (dsxRE) and a purine-rich element (PRE) at its 3' UTR same as did the 3' UTR of $M$. domestica and other Bactrocera insects. This suggests that the Btau-dsx female-specific splicing may also be regulated by the combined protein products of the two upstream genes, Btau-tra and Btau-tra2, which bind to the dsxREs and the PRE. Moreover, the copy number of the dsxRE domain seems to vary to as much as eight in Lucilia capurina (Concha et al., 2010); six in D. melanogaster (Inoue et al., 1992), Megaselia scalaris (Kuhn et al., 2000) and Anopheles gambiae (Scali et al., 2005); five in Aedes aegypti (Salvemini et al., 2011); four in M. domestica (Hediger et al., 2004), C. ceratitis (Saccone et al., 2008) and Bactrocera species (Shearman \& Frommer, 1998; Chen et al., 2008; Permpoon et al., 2011), and three in Anastrepha obligua (Ruiz et al., 2005). However, all Bactrocera $d s x$ female-specific transcripts including Btau- $d s x^{F}$ contain four dsxRE copies and one PRE domain. Therefore, the presence of dsxRE and PRE domains found in the Btau$d s x^{F}$ transcript also strongly suggests that the TRA/TRA2 protein complex is required for $d s x$ female-specific splicing in B. tau as with most dipteran insects.

\section{Expression of Btau-dsx}

In D. melanogaster, the $d s x$ gene is transcribed in both sexes but its pre-mRNA was spliced in sex specific manners, producing one $d s x$ male-specific mode in males and one female-specific mode in females. The $d s x$ male-specific transcript is the default state while the female-specific mode is a regulated state since the $d s x$ pre-mRNA spliced into the female-specific mode requires the activity of the TRA/TRA2 protein complex (Burtis \& Baker, 1989; Hedley \& Maniatis, 1991; Hoshijima et al., 1991; Inoue

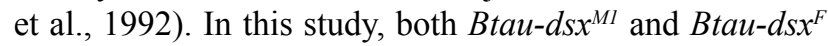
transcripts were detected in early fertilized eggs, but only $B t a u-d s x^{F}$ was detected in unfertilized eggs. The presence in unfertilized eggs of only the Btau-dsx $x^{F}$ transcript sug-

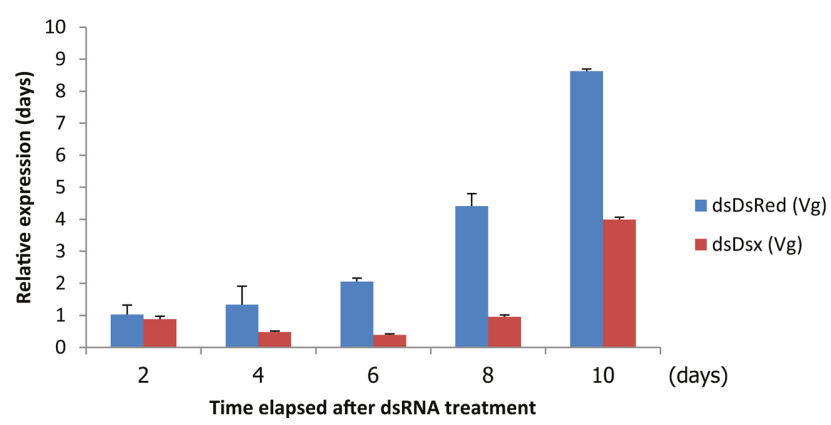

Fig. 9. Relative vitellogenin (Btau- $\mathrm{Vg}$ ) expression in females injected with either Btau-dsx dsRNA or DsRed dsRNA on 2, 4, 6, 8 and 10 days post-injection. Bars represent the mean $\pm S D$ obtained from qPCR experiment.

gests that Btau-dsx pre-mRNA may be a maternal substrate transferred to the eggs during oogenesis. Since we also detected the maternal transformer female-specific (Btau$\operatorname{tra}^{F}$ ) transcript (our unpublished data) and the maternal transformer2 (Btau-tra2) transcript (Thongsaiklaing et al., 2018) in early laid unfertilized and fertilized eggs, it is likely that in B. tau female embryos and in unfertilized eggs, the maternal Btau-dsx pre-mRNAs may be spliced into the female mode upon activation of the maternal BtauTRA/Btau-TRA2 protein complex. In early fertilized eggs,

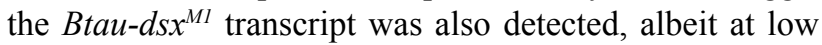
levels, indicating that the Btau- $d s x^{M 1}$ transcript may exist only in male embryos. In XY fertilized eggs of C. ceratitis, presence of the $C c d s x$ male-specific transcript suggested that the male determining $M$ factor on the $\mathrm{Y}$ chromosome could prevent maternal Cctra activity, thus leading to an absence of the CcTRA/CcTRA2 protein complex required for zygotic Cctra pre-mRNA splicing into the female mode via the positive feedback loop. As in D. melanogaster, in the absence of the female-specific $C_{c t r a} F$ transcript, the CcTRA/CcTRA2 protein complex could not form and thus $C c d s x$ was spliced by default to produce the CcDSX malespecific protein, which in turn induced male differentiation of XY embryos (Hoshijima et al., 1991; Pane et al., 2002; Salvemini et al., 2009). Moreover, the Btau- $d s x^{M 1}$ transcript appeared in early laid eggs $(0-0.5 \mathrm{~h})$, much earlier than in embryos of other insects such as $D$. melanogaster, $C$. ceratitis and B. jarvisi. In the former two insect species, the $d s x$ male-specific transcripts were first detected in male embryos $10 \mathrm{~h}$ after oviposition (Hempel \& Oliver, 2007; Gabrieli et al., 2010), while in the latter, the $d s x^{M}$ transcript was first detected in male embryos at $6 \mathrm{~h}$ after oviposition (Morrow et al., 2014). However, the expression of the $d s x$ male-specific transcript (Aadsx ${ }^{M}$ ) in $A$. aegypti was similar to that of $B t a u-d s x^{M 1}$ since it was detected in early laid eggs $(0.5 \mathrm{~h}$ after oviposition) and in the ovaries of adult females. The explanation for the presence of the Aads $x^{M}$ transcript in the adult ovaries was that the $A a d s x^{M}$ was not present in female tissues but presented in the fertilized eggs located within the ovaries of fertilized females (Salvemini et al., 2011).

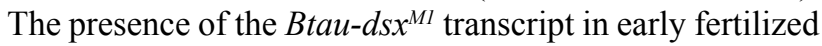
eggs suggests that the male determining $M$ factor on the $\mathrm{Y}$ chromosome may function at the early syncytial stage 
of XY fertilized eggs and that the M factor produced may function as a repressor to prevent the activity of maternal Btau-tra, resulting in the maternal Btau-TRA/Btau-TRA2 protein complex not being formed. Without this protein complex, the splicing pattern of the Btau-dsx pre-mRNA may be switched from the female (alternative) mode to the male (default) mode. In contrast, the Btau- $d s x^{M 2}$ transcript was first detected in adult males eighth days post-eclosion. Since the Btau- $d s x^{M 2}$ transcript encoded a truncated protein without the OD2 domain and sex-specific region, the Btau$\mathrm{DSX}^{\mathrm{M} 2}$ protein likely has no function, at least not in sex determination. Male $d s x$ transcripts without the OD2 domain were reported in $S$. coprophila and $S$. ocellaris but in these two insects, male and female $d s x$ transcripts were present in both sexes (Ruiz et al., 2015). However, Btau-DSX ${ }^{\mathrm{M} 2}$ contained the OD1 domain that functions as a DNA bind-

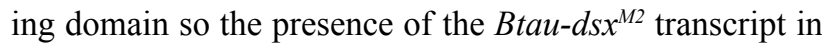
the adult stage could mean that this protein might be required for some other unknown function.

\section{Function of the Btau-dsx gene}

We examined the function of the Btau-dsx gene by injecting Btau-dsx dsRNA into the ventral side of the abdomens of just-emerged females, since this technique was quite suitable for examining the function and expression of genes, particularly genes expressed in the fat body such as vitellogenin $(\mathrm{Vg})$ genes (Amdam et al., 2003). The efficiency of this technique was confirmed in our experiment by comparing the levels of endogenous Btau-dsx $x^{F}$ mRNA in females injected with Btau-dsx dsRNA and females injected with DsRed dsRNA. We found that on all days posteclosion, Btau-dsx dsRNA injected females had lower levels of endogenous Btau-dsx mRNA than that of the DsRed dsRNA injected control females, particularly on the fourth day post-injection.

We also examined the level of Vitellogenin (Btau-Vg) mRNA in females injected with either Btau-dsx or DsRed dsRNAs since the expression of Vitellogenin was under the control of the female-specific DSX protein (Coschigano \& Wensink, 1993; Chen et al., 2008; Shukla \& Nagaraju, 2010). Vitellogenin ( Vg) is a precursor of the major yolk protein, an essential nutrient for the embryonic development of oviparous animals including insects (Valle, 1993). $\mathrm{Vg}$ is synthesized in the fat body and then accumulates in the eggs during oogenesis (Raikhel \& Dhadialla, 1992). In other insects, decreased expression of $V g$ genes affects the development of the oocytes, which in turn affects the morphology of ovaries (Chen et al., 2008; Shukla \& Nagaraju, 2010; Veerana et al., 2014). In this study, the ovaries dissected from females injected with Btau-dsx dsRNA showed only mild defects. In $B$. dorsalis, the ovaries of females injected with $2 \mu \mathrm{g} / \mu \mathrm{l}$ dsRNA per female were abnormal (Chen et al., 2008) and in Antheraea assama, ovaries dissected from females injected with $70 \mu \mathrm{g}$ of $\mathrm{Aads} x$ dsRNA per larva were deformed (Shukla \& Nagaraju, 2010). Therefore, it is likely that the concentration of Btau$d s x$ dsRNA ( $1 \mu \mathrm{g} / \mu \mathrm{l}$ per female) used in this study may not be enough to efficiently suppress expression of the Btau$d s x$ gene, such insufficient suppression would not lower
Btau- $V g$ expression levels under the threshold necessary to cause strong ovary defects.

In conclusion, the present study shows that the structure of the Btau-dsx gene is similar to that of most dipteran insects. In contrast to other tephritid orthologs, Btau-dsx produces three (one female and two male) instead of two (one female and one male) transcript variants. The novel malespecific protein (Btau-DSX ${ }^{\mathrm{M} 2}$ ) has no OD2 domain. The $B t a u-d s x^{M 2}$ is first detected in eight day-old adult males, indicating that Btau-dsx $x^{M 2}$ might have a role and is required only during the development of mature adult males, after sex has already been determined by $\mathrm{DSX}^{\mathrm{M} 1}$. Indeed, similar to A. aegypti $d s x$, Btau-dsx $x^{M 1}$ is detected at the early syncytium stage, but differs from the $d s x$ genes of most insects previously studied. The knowledge obtained from this study might be useful for developing advanced biotechnological methods to control the spread of $B$. tau and other insect pests, since sex determination genes can be used to develop transgenic sexing strains for male sterile insect techniques or pest management programmes using RNAi technology (Raphael et al., 2004; Dafa'alla et al., 2010; Fu et al., 2010; Whyard et al., 2015; Leftwich et al., 2016).

ACKNOWLEDGEMENTS. This work is supported by Kasetsart University Research and Development Institute (KURDY). We are grateful to N. Fascetti for her kind English checking and for providing suggestions on the manuscript. We thank the Higher Education Commission of Thailand, the Center of Agricultural Biotechnology, Kasetsart University, Kampaengsaen Campus, Nakon Pathom and the Center for Excellence on Agricultural Biotechnology: (AG-BIO/PERDO-CHE), Bangkok, Thailand, for the grant to TT.

\section{REFERENCES}

Amdam G.V., Simões Z.L.P., Guidugli K.R., NorberG K. \& OMHOLT S.W. 2003: Disruption of vitellogenin gene function in adult honeybees by intra-abdominal injection of double-stranded RNA. - BMC Biotechnol. 3: 1, 8 pp.

An W., Cho S., Ishi H. \& Wensink P.C. 1996: Sex-specific and non-sex-specific oligomerization domains in both of the doublesex transcription factors from Drosophila melanogaster. Mol. Cell Biol. 16: 3106-3111.

BAKER B.S. \& WolfNer M.F. 1988: A molecular analysis of doublesex, a bifunctional gene that controls both male and female sexual differentiation in Drosophila melanogaster. - Genes Dev. 2: 477-489.

Bopp D., Saccone G. \& Beye M. 2014: Sex determination in insects: Variations on a common theme. - Sex. Dev. 8: 20-28.

Burtis K.C. \& BAKER B.S. 1989: Drosophila doublesex gene controls somatic sexual differentiation by producing alternatively spliced mRNAs encoding related sex-specific polypeptides. Cell 56: 997-1010.

Chen S.C. 2001: The development and reproduction of pumpkin fly, Bactrocera tau (Walker) (Diptera: Tephritidae) and field occurrence investigation. — Plant Prot. Bull. Taichung. 43: 137-151.

Chen S.L., Dai S., Lu K. \& Chang C. 2008: Female-specific doublesex dsRNA interrupts yolk protein gene expression and reproductive ability in oriental fruit fly, Bactrocera dorsalis (Hendel). — Insect Biochem. Mol. Biol. 38: 155-165. 
Cho S., Huang Z. \& Zhang J. 2007: Sex-specific splicing of the honeybee doublesex gene reveals 300 million years of evolution at the bottom of the insect sex-determination pathway. Genetics 177: 1733-1741.

Cline T.W. 1993: The Drosophila sex determination signal: how do flies count to two? - Trends Genet. 9: 385-390.

Concha C., Li F. \& ScotT M.J. 2010: Conservation and sex-specific splicing of the doublesex gene in the economically important pest species Lucilia cuprina. - J. Genet. 89: 279-285.

Concha C. \& Scott M.J. 2009: Sexual development in Lucilia cuprina (Diptera, Calliphoridae) is controlled by the transformer gene. - Genetics 182: 785-798.

Coschigano K.T. \& Wensink P.C. 1993: Sex-specific transcriptional regulation by the male and female doublesex proteins of Drosophila. - Genes Dev. 7: 42-54.

Dafa'alla T., Fu G. \& Alphey L. 2010: Use of a regulatory mechanism of sex determination in pest insect control. $-J$. Genet. 89: 301-305.

ERdman S.E. \& BuRtis K.C. 1993: The Drosophila doublesex proteins share a novel zinc finger related DNA binding domain. - EMBO J. 12: 527-535.

Fu G., Lees R.S., Nimmo D., Aw D., Jin L., Gray P., Berendonk T., White-Cooper H., Scaife S., Kim Phuc H., Marinotti O., Jasinskiene N., James A. \& AlPhey L. 2010: Female-specific flightless phenotype for mosquito control. — Proc. Natl. Acad. Sci. 107: 4550-4554.

Gabrieli P., Falaguerra A., Siciliano P., Gomulski L.M., Scolari F., Zacharopoulou A., Franz G., Malacrida A.R. \& GasPERI G. 2010: Sex and the single embryo: early deveopment in the Mediterranean fruit fly, Ceratitis capitata. - BMC Dev. Biol. 10: 12, 14 pp.

Hediger M., Burghardt G., Siegenthaler C., Buser N., HilfiKER-Kleiner D., Dubendorfer A. \& Bopp D. 2004: Sex determination in Drosophila melanogaster and Musca domestica converges at the level of the terminal regulator doublesex. - Dev. Genes Evol. 214: 29-42.

Hedley M. \& Maniatis T. 1991: Sex-specific splicing and polyadenylation of $d s x$ pre-mRNA requires a sequence that binds specifically to tra-2 protein in vitro. - Cell 65: 579-586.

Hempel L.U. \& Oliver B. 2007: Sex-specific Doublesex (M) expression in subsets of Drosophila somatic gonad cells. - BMC Dev. Biol. 7: 113, 15 pp.

Hoshijima K., Inoue K., Higuchi I., SaKamoto H. \& Shimura Y. 1991: Control of doublesex alternative splicing by transformer and transformer-2 in Drosophila. - Science 252: 833-836.

Inoue K., Hoshijima K., Higuchi I., SaKamoto H. \& Shimura Y. 1992: Binding of the Drosophila transformer and transformer-2 proteins to the regulatory elements of doublesex primary transcript for sex-specific RNA processing. - Proc. Natl. Acad. Sci. U.S.A. 89: 8092-8096.

Ito Y., Harigai A., Nakata M., Hosoya T., Araya K., Oba Y., Ito A., Ohde T., Yaginuma T. \& NiImi T. 2013: The role of doublesex in the evolution of exaggerated horns in the Japanese rhinoceros beetle. - EMBO Rep. 14: 561-567.

Kuhn S., Sievert V. \& Traut W. 2000: The sex-determining gene doublesex in the fly Megaselia scalaris: conserved structure and sex-specific splicing. - Genome 43: 1011-1020.

Kumar S., Stecher G. \& Tamura K. 2016: MEGA7: Molecular Evolutionary Genetics Analysis Version 7.0 for Bigger Datasets. - Mol. Biol. Evol. 33: 1870-1874.

Lagos D., Ruiz M.F., Sánchez L. \& Komitopoulou K. 2005: Isolation and characterization of the Bactrocera oleae genes orthologous to the sex determining Sex-lethal and doublesex genes of Drosophila melanogaster. - Gene 384: 111-121.
Lagos D., Koukidou M., Savakis C. \& Komitopoulou K. 2007: The transformer gene in Bactrocera oleae: the genetic switch that determines its sex fate. - Insect Mol. Biol. 16: 221-230.

Leftwich P., Bolton M. \& Chapman T. 2016: Evolutionary biology and genetic techniques for insect control. - Evol. Appl. 9: $212-230$.

LivaK K.J. \& Schmittgen T.D. 2001: Analysis of relative gene expression data using real-time quantitative PCR and the 2(-Delta Delta C(T)) Method. - Methods 25: 402-408.

Morrow J., Riegler M., Frommer M. \& Shearman D. 2014: Expression patterns of sex-determination genes in single male and female embryos of two Bactrocera fruit fly species during early development. - Insect Mol. Biol. 23: 754-767.

Ohbayashi F., Suzuki M.G., Mita K., Okano K. \& Shimada T. 2001: A homologue of the Drosophila doublesex gene is transcribed into sex-specific mRNA isoforms in the silkworm, Bombyx mori. - Comp. Biochem. Physiol. (B) 128: 145-158.

Oliveira D.C.S.G., Werren J.H., Verhulst E.C., Giebel J.D., Kamping A., Beukeboom L.W. \& van de Zande L. 2009: Identification and characterization of the doublesex gene of Nasonia. - Insect Mol. Biol. 18: 315-324.

Pane A., Salvemini M., Bovi P.D., Polito C. \& Saccone G. 2002: The transformer gene in Ceratitis capitata provides a genetic basis for selecting and remembering the sexual fate. - Development 129: 3715-3725.

Permpoon R., Aketarawong N. \& Thanaphum S. 2011: Isolation and characterization of doublesex homologues in the Bactrocera species: B. dorsalis (Hendel) and B. correcta (Bezzi) and their putative promoter regulatory regions. - Genetica 139: 113-127.

Price D.C., Egizi A. \& Fonseca D.M. 2015: The ubiquity and ancestry of insect doublesex. - Sci. Rep. 5: 13068, 9 pp.

Raikhel A.S. \& Dhadialla T.S. 1992: Accumulation of yolk proteins in insect oocytes. - Annu. Rev. Entomol. 37: 217-251.

Raphael K.A., Whyard S., Shearman D., An X. \& Frommer M. 2004: Bactrocera tryoni and closely related pest tephritids-molecular analysis and prospects for transgenic control strategies. - Insect Biochem. Mol. Biol. 34: 167-176.

Ruiz M., Stefani R., Mascarenhas R., Perondini A., Selivon D. \& SÁNCHEZ L. 2005: The gene doublesex of the fruit fly Anastrepha obliqua (Diptera, Tephritidae). - Genetics 171: 849-854.

Ruiz M., Alvarez M., Eirin-Lopez J., Sarno F., Kremer L., BarBERO J. \& SANCHEZ L. 2015: An unusual role for doublesex in sex determination in the dipteran Sciara. - Genetics 200: 1181-1199.

Saccone G., Peluso I., Artiaco D., Giordano E., Bopp D. \& Polito L.C. 1998: The Ceratitis capitata homologue of the Drosophila sex-determining gene Sex-lethal is structurally conserved, but not sex-specifically regulated. - Development 125: $1495-1500$.

Saccone G., Salvemini M., Pane A. \& Polito L.C. 2008: Masculinization of XX Drosophila transgenic flies expressing the Ceratitis capitata DoublesexM isoform. - Int. J. Dev. Biol. 52: 1051-1057.

Salvemini M., Robertson M., Aronson B., Atkinson P., Polito C. $\&$ SACCONE G. 2009: Ceratitis capitata transformer-2 gene is required to establish and maintain the autoregulation of Cctra, the master gene for female sex determination. - Int. J. Dev. Biol. 53: 109-120.

Salvemini M., Mauro U., Lombardo F., Milano A., Zazzaro V., Arcà B., Polito L. \& Saccone G. 2011: Genomic organization and splicing evolution of the doublesex gene, a Drosophila regulator of sexual differentiation, in the dengue and yellow fever mosquito Aedes aegypti. — BMC Evol. Biol. 11: 41, 19 pp. 
Salz H.K. \& ERICKSon J.W. 2010: Sex determination in Drosophila: the view from the top. - Fly 4: 60-70.

Sarno F., Ruiz M.F., Eirín-López J.M., Perondini A.L.P., Selivon D. \& SÁNCHEZ L. 2010: The gene transformer-2 of Anastrepha fruit flies (Diptera, Tephritidae) and its evolution in insects. BMC Evol. Biol. 10: 140, 13 pp.

Scali C., Catteruccia F., Li Q. \& Crisanti A. 2005: Identification of sex-specific transcripts of the Anopheles gambiae doublesex gene. - J. Exp. Biol. 208: 3701-3709.

SheARMAn D.C.A. \& Frommer M. 1998: The Bactrocera tryoni homologue of the Drosophila melanogaster sex-determination gene doublesex. - Insect Mol. Biol. 7: 355-366.

ShuKLa J.N. \& Nagaraju J. 2010: Two female-specific DSX proteins are encoded by the sex-specific transcripts of dsx, and are required for female sexual differentiation in two wild silkmoth species, Antheraea assama and Antheraea mylitta (Lepidoptera, Saturniidae). - Insect Biochem. Mol. Biol. 40: 672-682.

ShUKLA J.N. \& PAlli S.R. 2012: Sex determination in beetles: production of all male progeny by parental RNAi knockdown of transformer. - Sci. Rep. 2: 602, 9 pp.

Thongsaiklaing T., Nipitwattanaphon M. \& NGernsiRi L. 2018: The transformer 2 gene of the pumpkin fruit fly, Bactrocera tau (Walker), functions in sex determination, male fertility and testis development. - Insect Mol. Biol. doi:10.1111/imb.12517

VALLE D. 1993: Vitellogenesis in insects and other groups - a review. - Mem. Inst. Oswaldo Cruz 88: 1-26.

Veerana M., Kubera A. \& Ngernsiri L. 2014: Analysis of the Vitellogenin gene of rice moth, Corcyra cephalonica Stainton. - Arch. Insect Biochem. Physiol. 87: 126-147.

Verhulst E., van de Zande L. \& Beukeboom L. 2010: Insect sex determination: it all evolves around transformer. - Curr. Opin. Genet. Dev. 20: 376-383.

Whyard S., Erdelyan C.N.G., Partridge A.L., Singh A.D., Beebe N.W. \& CAPINA R. 2015: Silencing the buzz: a new approach to population suppression of mosquitoes by feeding larvae double-stranded RNAs. - Parasit. Vectors 8: 96, 11 pp.

Received May 2, 2018; revised and accepted September 7, 2018 Published online October 24, 2018
Table S1. Doublesex proteins of a selection of insect species available on the NCBI database used for alignment. Supplementary data Spreadsheet containing the raw data obtained from qPCR analysis for Btau-dsx and Btau-Vg expression levels, comparing adult females injected with the Btau-dsx dsRNA to adult females injected with DsRed dsRNA, examined at 2, 4, 6, 8 and 10 days post-injection. The results for detecting the expression of $d s x$ and $V g$ are displayed in Figs 8 and 9, respectively.

\begin{tabular}{lcc}
\hline Insect species & \multicolumn{2}{c}{ Accession numbers } \\
\hline Aedes aegypti & ABD96571.1 & ABD96573.2 \\
Anastrepha obliqua & AAY25166 & AAY25168 \\
Anastrepha amita & ABF50961 & ABF50951 \\
Anastrepha bistrigata & ABF50960 & ABF50950 \\
Anastrepha fraterculus & ABF50953 & ABF50943 \\
Anopheles gambiae & AIY68269.2 & AIY68268.3 \\
Antheraea mylitta & ADL40853.1 & ADL40855.2 \\
Apis mellifera & ABV55180.1 & ABW99102.2 \\
Bactrocera correcta & ACN73403 & ACN73403 \\
Bactrocera dorsalis & AAV85891 & AAV85891 \\
Bactrocera jarvisi & AlK66583 & KJ816790 \\
Bactrocera oleae & AJ547621 & CAD67988 \\
Bactrocera tryoni & AAB99948 & AAB99948 \\
Ceratitis capitata & AAN63598 & AAN63598 \\
Drosophila melanogaster & NP_731198 & NP_731199 \\
Helicoverpa armigera & AHF81652.1 & AHF81649.2 \\
Lucilia cuprina & ADG37649.1 & ADG37648.2 \\
Megaselia scalaris & AAK38831.1 & AAK38832.2 \\
Musca domestica & AAR23812.1 & AAR23813.2 \\
Nasonia vitripennis & ACJ65507.1 & ACJ65510.2 \\
Onthophagus taurus & AEX92939.1 & AEX92938.2 \\
Tribolium castaneum & AFQ62106.1 & AFQ62105.2 \\
\hline
\end{tabular}

